\title{
Development of a digital photo hoarding scale: A research with undergraduate students
}

\author{
İbrahim Bozacl $^{\mathbf{a}^{*}}$ and İsmail Gökdeniz ${ }^{\mathrm{a}}$
}

${ }^{a}$ Kırlkkale University, Keskin Vocational High School, Department of Marketing, Kırșehir Road, 3. km, 71800, Keskin/Kırıkkale, Turkey ${ }^{b}$ Kırlkkale University, Faculty of Economics and Administrative Sciences, Department of Business, Ankara Road, 7. km, Yahsihan/Kırıkkale, Turkey

\section{CH R O N I C L E}

\section{Article history:}

Received: February 15, 2020

Received in revised format:

March 162020

Accepted: March 16, 2020

Available online:

March 16, 2020

Keywords:

Hoarding

Digital hoarding

Hoarding of digital photo

Addiction, consumer

\begin{abstract}
A B S T R A C T
This study focuses on hoarding of digital assets. Today people's ownership of digital assets can be uncontrolled and the measurement tool designed in the study is expected to be useful for young people, health care organizations, businesses (smartphone firms etc.) and researchers. In the study, previous researches on hoarding, in particular hoarding of digital assets are reviewed. We then describe the process by which we developed our digital photograph hoarding scale (DPHS): development of scale items, evaluation of items, testing of a preliminary version, conducting validity and reliability analyses and analysis of scale scores. As a result, sub-dimensions of the digital photograph hoarding are identified as: problems caused by uncontrolled acquisition of photographs; problems caused by clutter; uncontrolled clutter of photographs; failure to dispose of photographs and related problems; uncontrolled taking of photographs accompanied by a constant desire to do so. It is seen that people with high DPHS scores also have higher scores on measures of photographing and photograph examination. Finally, the limitations of the research are discussed and suggestions for the future researches are offered.
\end{abstract}

C 2020 by the authors; licensee Growing Science, Canada

\section{Introduction}

Various definitions of hoarding have been offered; it has been described as the acquisition of assets that are useless or of limited value and failure to dispose of them (Frost \& Gross, 1993) and as continuous difficulty in disposing of assets due to a perceived need to accumulate, excessive accumulation of assets and severe clutter, characterized by stress and damage to life functions caused by assets (Frost \& Hartl, 1996). When having excessive assets (products, etc.) has negative consequences it can be recognized as a health problem, under the term 'hoarding'. Pathological hoarding, which affects everyday life functions and is related to a desire for protection, is examined in psychological and psychiatric research (Stiff et al., 1975). Hoarding of concrete objects such as books, magazines, newspapers, clothes and furniture has been the subject of research interest for some time, but the hoarding of digital assets is a relatively new research area. There have been few studies of the problems associated with ownership and use of digital assets, although this is difficult and can cause behavioral problems. Hoarding is a fundamental behavioral and social problem that must be managed because it is a waste of time, causes problems for people and adversely affects their professional and social lives. Qualitative research and case studies of digital hoarding have drawn attention to the problem. The development of a scale of digital hoarding will enable identification of digital hoarding and its scope and facilitate a more systematic understanding of the problem.

\section{Measurement of Hoarding}

One of the first measures of hoarding of concrete assets to be developed is the Hoarding Scale (Frost \& Gross, 1993, 1996). The scale contains items on difficulty in disposing of assets, emotional reactions to disposal, problems in deciding on disposal, 
irregular use of accumulated assets, concern about the future use of assets that have been disposed of and emotional commitment to assets (Frost \& Gross, 1993). However, the scale was criticized its creators because it does not capture symptoms such as over-acquisition, some items refer to beliefs rather than behavior and it does not fully capture stress and problems caused by asset accumulation. This led to the development of a new scale that addressed these deficiencies. The Saving Inventory-Revised (Frost et al., 2004), which is widely used, measures several aspects of hoarding: difficulty in disposal, excessive clutter and excessive acquisition. It has been recognized that the SI-R is effective in distinguishing hoarders from non-hoarders (Frost et al., 2004). Another commonly used tool in the measurement of hoarding is the Yale-Brown ObsessiveCompulsive Scale (Y-BOCS). The Y-BOCS evaluates several aspects of hoarding: frequency, the associated stress, deterioration in social and professional life, resistance and control of situations related to obsessions, but because responses are given using a dichotomous (yes-no) scale it does not provide any indication of the scope and severity of a hoarding problem (Frost \& Hartl, 1996). The Hoarding Rating Scale (Tolin et al., 2010) is another measurement tool used to identify hoarding. It consists of questions about difficulty using rooms due to the accumulation of clutter, difficulty in disposing of assets, problems caused by obtaining more products than necessary, affordable or useful, the emotional stress caused by clutter, acquisition of clutter and the deteriorations in life resulting from hoarding behavior (effects on daily routine, work or school life, social activities, family life, financial situation etc.). Responses are given using a scale ranging from 0 to 8 . Several studies have confirmed the reliability and validity of the HRS and it has been shown to be strongly correlated with related measures (Tolin et al., 2010; Tolin et al., 2018).

Guillard and Pinson (2012) measured consumers' hoarding tendency using the following items: 'I usually tend to keep assets', 'I'm someone who likes to keep products rather than dispose of them' and 'I would like to keep everything if I could'. They distinguish between instrumental motives for hoarding (thoughts such as 'I might need it one day'), emotional motives ('it reminds me of things I did'), social motives ('it might be useful to someone') and economic motives (throwing things away feels like a waste of money) (Guillard \& Pinson, 2012). Guillard and Pinson (2012) focused on what people think and feel about the disposal of assets; their measure does not cover other dimensions of hoarding and focuses on the motives leading to hoarding.

There are various other tools such as the 10-item UCLA Hoarding Severity Scale (UHSS), which is generally used in clinical cases; it was based on previous research and covers level of clutter, accumulation impulses, excessive acquisition, difficulty in disposal, adverse effects on social and professional life (Saxena, Brody, Maidment, \& Baxter, 2007); the Hoarding Assessment Scale (HAS), which covers difficulty of disposal, clutter, accumulation impulses and problems and stress related to hoarding (Schneider, Storch, Geffken, Lack, \& Shytle, 2008); the Saving Cognitions Inventory (SCI), which captures emotional commitment to assets, beliefs about memories, control and responsibility (Steketee et al., 2003; Frost et al., 2004) and the Compulsive Acquisition Scale (CAS), which assesses compulsive shopping and compulsive acquisition of free products (Frost, Tolin, Steketee, Fitch, \& Selbo-Bruns, 2009).

\section{Hoarding of Digital Assets: Causes and Results}

Hoarding, which has traditionally been described as the obsessive accumulation of tangible objects (in some cases most of the room in which the individual lives is filled with books and magazines), has recently attracted attention as a phenomenon affecting digital products (Fried, 2014). Van Bennekom et al. (2015) described the hoarding of digital assets as "uncontrolled accumulation of digital files to cause irregularity and stress", which is similar to descriptions of hoarding of tangible assets. In their study, they argue that digital hoarding is a problem that needs to be detected and treated. Individuals can hoard digital pictures to such an extent that it has a negative impact on their daily life (they may take a thousand pictures a day). Hoarders of digital photographs do not delete similar images, may never look at them, yet convince themselves that they will use them in the future. In one clinical case the individual experienced feelings of nervousness and loss of time due to the hoarding of digital images; this individual was devoting 35 hours per day to taking photographs and the digital hoarding was having adverse effects on the individual's sleeping pattern and other activities such as house cleaning, walking and relaxation (Van Bennekom et al., 2015).

Digital hoarding can be influenced by factors such as the thought that one may need the data in the future, laziness, lack of motivation to manage digital files, time constraints and emotional commitment to the data (Sweeten, Sillence, \& Neave, 2018), the development of digital storage capacity (Schull, 2018), the existence of tools for creating content (communication applications, smartphones, etc.), natural motives for creativity such as the desire to share ideas (Williams, Leighton John, \& Rowland, 2009), not knowing which data are needed, fear of losing data and not knowing what to do with data (Gormley \& Gormley, 2012), creation of evidence, sharing of resources, identity creation and 'just in case' motivation (Kaye et al., 2006).

Problems caused by digital data hoarding are: decreased productivity, lower psychological well-being (creating stress, nervousness, misery, etc.) cybersecurity problems (Sweeten et al., 2018), increase in costs (it has been claimed that US service providers and data centers account for a higher percentage of total national electricity consumption than any other sector) (Sloane, 2011), the loss of data value, time lost by employees searching for data and a culture of data hoarding in businesses that prevents them from benefiting from useful data (Gormley \& Gormley, 2012). In short, the adverse effects of digital hoarding spread wider than the hoarders themselves, affecting the institutions where they work and society in general. 


\section{Development of a Digital Hoarding Scale for Photographic Assets: Research with Undergraduate Students}

The aim of this part of the research was to develop a scale for the digital assets. We started by creating a pool of potential items and analysing their scope. We then piloted a preliminary version of the scale in a sample of 20 students to assess the comprehensibility of the items. A revised version of the scale was then tested in a sample of 458 undergraduate students; the Kaiser-Meyer-Olkin score and Bartlett's sphericity test were applied to the data, which were subsequently subjected to exploratory factor analysis, item analysis (item-total correlation, item residual correlation, item discrimination) and reliability analysis (assessment of internal consistency and split-half reliability).

\subsection{Preliminary Research on the Type of Digital Asset Hoarded}

We surveyed 312 students of the Faculty of Economics and Administrative Sciences, Kırıkkale University to determine which digital assets were most frequently hoarded. Students were asked questions about what type of digital assets they acquired most frequently, found themselves unable to delete and accumulated as digital clutter. This preliminary research suggested that the most commonly hoarded digital assets are photographs, music and video clips. The assets most commonly cited as most frequently acquired by our student sample were photographs $(\mathrm{n}=154)$ and music $(\mathrm{n}=131)$. Failure to delete was most common for photographs $(\mathrm{n}=117)$ and videos $(\mathrm{n}=107)$. Photographs were the asset most commonly cited as the leading contributor to digital clutter $(n=136)$ followed by music $(n=116)$. Based on these data, we decided to develop a scale to measure the digital hoarding of photographs.

\subsection{Scale Development}

Based on existing scales of hoarding and the definitions of digital hoarding in the literature we decided to create a scale that would measure the following main dimensions of hoarding: uncontrolled acquisition, failure to dispose of assets, extent of clutter and the resulting problems. We generated a pool of 36 items covering these dimensions.

Consulting a panel of experts is generally the preferred method of evaluating whether the scope of a scale or pool of items is appropriate (Yurdugul, 2005). We therefore asked five researchers in the field of marketing to evaluate whether our pool of items captured the phenomenon of hoarding of digital assets, whether the content of items was appropriate and whether there were any items that were too similar to each other. We excluded items deemed by our experts to be duplicates were excluded, leaving a 22-item questionnaire using a five-point Likert scale format with response ranging from 1 (I strongly disagree) to 5 (I strongly agree). The clarity of the items and the existence of typographical errors were evaluated in a sample of 20 students.

\subsection{Testing of the Scale}

\subsubsection{Sample}

The scale was tested in a sample of 458 students in the Faculty of Economics and Administrative Sciences, Kirikkale University. As the number of cases required for a meaningful and reliable analysis is at least ten times the number of questions (Tavsancil, 2014; MacCallum et al., 1999), our sample size was sufficient. About a third of the sample $(n=143,32.1 \%)$ was male and $67.9 \%(n=303)$ was female. The age distribution was as follows: $\leq 19$ years $(4.5 \%, n=20) ; 20-22$ years $(69.1 \%$, $n=308) ; \geq 23$ years $(26.5 \%)$.

\subsubsection{Validity Analysis}

Exploratory factor analysis and item analyses were used to characterize the structure of the questionnaire. Conducting confirmatory factor analysis is not recommended on the same sample and data on a newly developed scale (Erkus, 2012). Briefly, factor analysis has been used to determine the structure of a data set, specifically the implicit variables, or factors underlying a group of expressions (Devellis, 2014). The Kaiser-Meyer-Olkin test and Bartlett's test of sphericity were used to determine whether the data were suitable for factor analysis. The KMO value indicates whether a sample is adequate and should be greater than 0.5. Bartlett's sphericity test determines whether a scale's items are related to each other and whether the scale consists of one or more dimensions; probability values of is less than .05 indicate that the scale is effective in measuring the sub-dimensions of the phenomenon under investigation (Ozdamar, 2016). Our data had a KMO value of .882 and Bartlett test's chi-squared was significant, indicating that the data were suitable for factor analysis and had a multivariate normal distribution.

In the second stage of the analysis we examined the factor structure of the data and the percentage of variance in overall score accounted for by each factor. Five factors explained $76.176 \%$ of the total variance, which is considered sufficient, since a measure which accounts for $40 \%$ to $60 \%$ of variance in the phenomenon of interest is considered as adequate in the social sciences (Acherer, Wiebe, Luther, \& Adams, 1988). Components with an eigenvalue higher than one are taken into consideration. The factor values are shown in Table 1. After examining the percentages of variance explained by the factors, we investigated the distribution of items across factors by performing varimax vertical rotation. It was considered that there should be at least 0.10 difference between the factors that have a significant load on more than one factor (Tavsancil, 2014). The item 'Disposing of digital images (e.g. deleting them) has an adverse effect on my life' loaded similarly on the first and fourth factors (.568 and .571 respectively) so this item was removed from the scale, leaving 21 items. 
Table 1

Exploratory Factor Analysis

\begin{tabular}{lllllll}
\hline \multirow{2}{*}{ Component } & \multicolumn{2}{l}{ Initial Eigen values } & \multicolumn{3}{c}{ Rotation Sums of Squared Loadings } \\
\cline { 2 - 7 } & Total & \% of Variance & Cumulative \% & Total & \% of Variance \\
\hline 1 & 9.422 & 42.828 & 42.828 & 5.513 & 25.059 & Cumulative \% \\
2 & 2,612 & 11.870 & 54.698 & 3,402 & 15.462 & 40.521 \\
3 & 1,982 & 9.011 & 63.709 & 3,036 & 13.800 & 54.321 \\
4 & 1,565 & 7.115 & 70.824 & 2,591 & 11.776 & 65.097 \\
5 & 1,177 & 5.351 & 76.176 & 2,217 & 10.079 & 76.176 \\
\hline
\end{tabular}

The factors were labelled as follows, factor 1: 'problems caused by the uncontrolled acquisition of photographs', factor 2: 'problems caused by clutter', factor 3: 'uncontrolled clutter of photographs', factor 4: 'failure to dispose of photographs and related problems' and factor 5: 'uncontrolled taking of photographs accompanied by a constant desire to do so'.

Table 2

Rotated Components Matrix

\begin{tabular}{|c|c|}
\hline & Factor Loadings \\
\hline \multicolumn{2}{|l|}{ Factor 1: Problems caused by the uncontrolled acquisition of photographs } \\
\hline My life is adversely affected by looking for new photographic opportunities in an uncontrolled manner & .863 \\
\hline My life is negatively affected when I don't take new photographs & .826 \\
\hline Uncontrollable searching for new photographic opportunities causes me stress & .808 \\
\hline My life (work, social, health, etc.) is negatively affected by my uncontrolled taking of photographs & .792 \\
\hline Taking new photographs in an uncontrolled way causes me stress & .780 \\
\hline Not taking new photographs causes me stress & .742 \\
\hline Failure to dispose of photographs negatively affects my life (work, social, etc.) & 689 \\
\hline \multicolumn{2}{|l|}{ Factor 2: Problems caused by clutter } \\
\hline My clutter causes me stress & .847 \\
\hline My problems controlling my clutter are stressful for me & .816 \\
\hline It is difficult for me to access photographs as I want to because of the mess and this is stressful & .779 \\
\hline My clutter adversely affects my daily life (work, social etc.) & .731 \\
\hline I don't use most of my photographs & .535 \\
\hline \multicolumn{2}{|l|}{ Factor 3: Uncontrolled clutter of photographs } \\
\hline The way my photographs are stored is extremely messy & .902 \\
\hline I have difficulty managing the clutter of my photographs & .871 \\
\hline It is hard to find the photographs I want because of the mess & .854 \\
\hline \multicolumn{2}{|l|}{ Factor 4: Failure to dispose of photographs and related problems (Even though I don't use the photographs) } \\
\hline I store my photographs on digital media (computer, flash memory, hard disk etc.) & .808 \\
\hline I have more problem disposing of digital images than most people & .785 \\
\hline I find disposing of photographs stressful & .657 \\
\hline Disposing of photographs adversely affects my life (work, social etc.) & .571 (loading on factor $1: .568$ ) \\
\hline \multicolumn{2}{|l|}{ Factor 5: Taking photographs in an uncontrolled manner and the constant desire to take photographs } \\
\hline I can't control my photo-taking behavior & .827 \\
\hline I always want to take new photographs & .777 \\
\hline I'm always looking for opportunities to take photographs, no matter where I am or what I'm doing & 683 \\
\hline
\end{tabular}

\subsubsection{Item Analysis and Reliability Analysis}

Item analysis provides information about the structural validity of a scale and is used to ensure that scales are internally consistent. Item-total correlations should be at least .20 for all items. The item-remainder correlation is the correlation between a given item score and the total scale score excluding this item and values should be at least .20 or .25 . Items with inadequate item-total or item-remainder correlations should be excluded from the scale (Tavsancil, 2014; Erkus, 2012). We calculated item-total and item-remainder correlations for all items the lowest values were .428 and .369 respectively and so no items were removed on the basis of these analyses.

Item discrimination analysis involves comparing the top $27 \%$ and bottom $27 \%$ of the sample (with respect to total score). If the difference between the mean scores of the upper and lower groups on a given item is significant then that item is considered to discriminate between high and scorers (Tavsancil, 2014). We concluded that all the items of our scale discriminated between hoarders and non-hoarders and therefore retained them all. The results of the item discrimination analysis are shown in Table 3.

The alpha coefficient provides a measure of the homogeneity of the items of a scale and whether they form a whole. If the alpha coefficient for a scale increases by more than $5 \%$ when an item is removed the item should be discarded (Ozdamar, 2016). In our sample our scale had a Cronbach's alpha coefficient of .923, Guttman's split-half reliability value was .745 and the Spearman-Brown split-half reliability coefficient was .746. The internal consistency coefficients for the factors were as 
follows, factor 1: .930; factor 2: .889; factor 3: .925; factor 4: .798; factor 5: .773. None of the item exclusions produced a significant increase in reliability and the scale were evaluated as reliable.

Table 3

Item Analysis

\begin{tabular}{|c|c|c|c|c|c|c|}
\hline & Item-Total correlation & $p$ & Item-Remainder correlation & $P$ & Item Discrimination & $p$ \\
\hline i1 & .428 & .000 & .369 & .000 & 8.412 & .000 \\
\hline $\mathrm{i} 2$ & .544 & .000 & .487 & .000 & 12.356 & .000 \\
\hline i3 & .483 & .000 & .420 & .000 & 10.835 & .000 \\
\hline $\mathrm{i} 4$ & .641 & .000 & .602 & .000 & 16.556 & .000 \\
\hline i5 & .667 & .000 & .631 & .000 & 15.915 & .000 \\
\hline i6 & .641 & .000 & .603 & .000 & 12.954 & .000 \\
\hline i7 & .713 & .000 & .682 & .000 & 17.786 & .000 \\
\hline i8 & .731 & .000 & .704 & .000 & 17.901 & .000 \\
\hline i9 & .645 & .000 & .611 & .000 & 12.449 & .000 \\
\hline $\mathrm{i} 10$ & .442 & .000 & .377 & .000 & 9.365 & .000 \\
\hline i11 & .626 & .000 & .576 & .000 & 16.646 & .000 \\
\hline $\mathrm{i} 12$ & .765 & .000 & .732 & .000 & 22.088 & .000 \\
\hline i14 & .754 & .000 & .724 & .000 & 18.178 & .000 \\
\hline $\mathrm{i} 15$ & .571 & .000 & .516 & .000 & 13.819 & .000 \\
\hline i16 & .652 & .000 & .607 & .000 & 17.938 & .000 \\
\hline i17 & .631 & .000 & .583 & .000 & 16.819 & .000 \\
\hline i18 & .547 & .000 & .495 & .000 & 11.918 & .000 \\
\hline i19 & .632 & .000 & .585 & .000 & 15.433 & .000 \\
\hline i20 & .744 & .000 & .711 & .000 & 23.221 & .000 \\
\hline i21 & .737 & .000 & .704 & .000 & 21.136 & .000 \\
\hline i22 & .725 & .000 & .688 & .000 & 22.581 & .000 \\
\hline
\end{tabular}

\subsubsection{Evaluation of Scale Scores}

Evaluation of subscale scores revealed that the subscale with the highest average was 'taking photographs in an uncontrolled manner and the constant desire to take photographs', whereas the subscale with the lowest average was 'the problems caused by uncontrolled photograph acquisition'. The average was between 1.93 and 2.77 (within 1-5 points), indicating that the problem is not common.

Table 4

Factor Averages

\begin{tabular}{lll}
\hline & Average & SD \\
\hline Problems caused by the uncontrolled acquisition of photographs & 1.9255 & .87389 \\
Problems caused by clutter & 2.3850 & .98955 \\
Uncontrolled clutter of photographs & 2.5373 & 1.18582 \\
Failure to dispose of photographs and problems caused by this & 2.7720 & 1.07385 \\
Taking photographs in an uncontrolled manner and the constant desire to take photographs & 2.7734 & 1.05192
\end{tabular}

Table 5

Allocation of digital hoarding subscales by group

\begin{tabular}{|c|c|c|c|}
\hline & Average & $\mathbf{Q}$ & $\%$ \\
\hline \multirow{3}{*}{ Problems caused by the uncontrolled acquisition of photographs } & $0-2.99$ & 394 & 86.8 \\
\hline & $3-3.99$ & 46 & 10.1 \\
\hline & $\geq 4$ & 14 & 3.1 \\
\hline \multirow{3}{*}{ Problems caused by clutter } & $0-2.99$ & 306 & 67.4 \\
\hline & $3-3.99$ & 100 & 22.0 \\
\hline & $\geq 4$ & 48 & 10.6 \\
\hline \multirow{3}{*}{ Uncontrolled clutter of photographs } & $0-2.99$ & 278 & 61.0 \\
\hline & $3-3.99$ & 90 & 19.7 \\
\hline & $\geq 4$ & 88 & 19.3 \\
\hline \multirow{3}{*}{ Failure to dispose of photographs and problems caused by this } & $0-2.99$ & 244 & 54.7 \\
\hline & $3-3.99$ & 126 & 28.3 \\
\hline & $\geq 4$ & 76 & 17.0 \\
\hline \multirow{3}{*}{ Taking photographs in an uncontrolled manner and the constant desire to take photographs } & $0-2.99$ & 268 & 58.8 \\
\hline & $3-3.99$ & 96 & 21.1 \\
\hline & $\geq 4$ & 92 & 20.2 \\
\hline
\end{tabular}


To determine the characteristics of people who display problematic hoarding of digital photographs we started by grouping scores into categories: 1-2.99 (low); 3-3.99 (medium); 4-5 (high). Based on these categories 3.1\% of people had high scores for 'problems caused by uncontrolled acquisition of photographs', $10.6 \%$ for 'problems caused by clutter', $19.3 \%$ for 'uncontrolled clutter of photographs', $17 \%$ for 'failure to dispose of photographs and problems caused by this' and $20.2 \%$ for 'taking photographs in an uncontrolled manner and the constant desire to take photographs'. In addition, similar distributions were examined depending on the total scores obtained from the scale. The maximum possible score on the final 21-item version of the scale is 105 and high scores indicate problematic hoarding. If scores of $\geq 84$ (representing an item average of $\geq 4$ ) are taken as an indication of problematic hoarding then $3.5 \%$ of our sample can be classified as problematic hoarders of digital photographs. Similarly, 3.5\% of the samples had scores more than 2 standard deviations above the mean and $15.3 \%$ had scores more than 1 standard deviation above the mean.

\subsubsection{Investigation of Differences in Photographing Behaviors between Hoarders and Non-Hoarders}

We compared the photographing habits of respondents with total scores more than 1SD above the mean (hoarders) or more than 1SD below the mean (non-hoarders). Hoarders used more smartphone photographs per day, spent more time taking pictures, spend more time looking at photographs and had taken more unviewed smartphone photographs in the last month. These differences were statistically significant. This suggests that our digital photograph hoarding scale predicts concrete hoarding behaviors.

Table 6

Relationship between Photographing Behaviors and the Scale Scores

\begin{tabular}{|c|c|c|c|}
\hline & & Average & Sig. \\
\hline \multirow[b]{2}{*}{ Number of smartphone photographs taken per day } & Non-hoarders & 5.72 & \multirow{2}{*}{0.002} \\
\hline & Hoarders & 13.69 & \\
\hline \multirow[b]{2}{*}{ Time spent taking photograph per day } & Non-hoarders & $11.16 \mathrm{~min}$. & \multirow{2}{*}{0.000} \\
\hline & Hoarders & $26.94 \mathrm{~min}$. & \\
\hline \multirow[b]{2}{*}{ Time spent reviewing photographs per day } & Non-hoarders & $17.07 \mathrm{~min}$. & \multirow{2}{*}{0.000} \\
\hline & Hoarders & $38.19 \mathrm{~min}$. & \\
\hline \multirow[b]{2}{*}{ Number of unviewed smartphone photographs taken during the last month } & Non-hoarders & 393 & \multirow{2}{*}{0.014} \\
\hline & Hoarders & 585 & \\
\hline
\end{tabular}

\section{Conclusion and discussion}

In this study we have pointed out that digital assets can be hoarded and presented a scale for measuring hoarding of digital photographs. To determine the dimensions of digital hoarding we started by reviewing tools used to evaluate the hoarding of material assets and based on this we concluded that the main dimensions of hoarding are uncontrolled acquisition of assets, failure to dispose of them, accumulation of clutter and the problems caused by this. We then created a pool of items designed to assess digital hoarding; these were subsequently reviewed by experts in the field of consumer behavior and questions which were deemed not to measure digital hoarding were removed, thereby improving the scope and validity of the scale. The resulting preliminary scale was piloted in a sample of 20 undergraduate students and formal and semantic errors were corrected. The reliability and validity of the revised scale were then tested in a sample of 458 undergraduate students and the scale was finalized.

We used exploratory factor analysis to assess the structural validity of the scale and this identified five dimensions of digital photograph hoarding: 'problems caused by uncontrolled acquisition of photograph', 'problems caused by clutter', 'uncontrolled clutter of photographs', 'failure to dispose of photographs and problems caused by this' and 'taking photographs in an uncontrolled manner and the constant desire to take photographs'. Together these factors accounted for $76.2 \%$ of the variance in scores on our digital photograph hoarding scale. Analyses of item-total correlations, item remainder correlations and item discrimination also supported the validity of the scale.

Analysis of respondents' total scores showed that $16(3.5 \%)$ had scores at least two standard deviations above the mean and the same number had scores $\geq 84$ (representing an overall average of $\geq 4 ; 4 \times 21=84$ ). Seventy respondents $(15.3 \%)$ had total scores at least one standard deviation above the mean. These figures give an indication of the prevalence of digital photograph hoarding.

We also found that those with total scores at least one standard deviation above the mean also had higher than average scores for number of smartphone photographs taken per day, amount of time spent taking pictures per day, time spent reviewing photographs per day and number of unviewed smartphone photographs taken during the last month. We infer from this that scores on our digital photography hoarding scale reflect photographing and examination of photographs and that the scale is suitable for measuring the hoarding of digital photographs.

This research has some limitations. First of all, we relied on self-report data and we have to assume that participants gave honest responses to questionnaire items. It is possible, however, for people to misrepresent the extent of uncontrolled and 
excessive thoughts, feelings and behaviors. It would be interesting to compare the results of our study with findings obtained using alternative methods such as neurological research methods and 'clutter image rating'. For example, by using clutter image rating, C-I-R scale developed in determining the hoarding symptoms, individuals are shown nine similar images and asked which of them best represents their own rooms. It has been claimed that this measure give similar results with other measures (Frost et al., 2008). It would be possible to develop similar methods for digital assets and compare them with our hoarding scale.

Adapting the scale to measure hoarding of other digital assets (video, music, etc.) and testing on individuals with different demographic characteristics will increase the applicability of the scale. Development of tool for measuring various forms of digital hoarding will make it easier to identify problematic behaviors that people exhibit in relation to digital assets.

The results of our research may be useful to marketing professionals. Firms, consumers and society in general could benefit from offering digital hoarding management mobile applications, drawing consumers' attention to digital hoarding, placing more emphasis on features of a product other than its capacity to take and store large numbers of photographs, supporting social campaigns about digital hoarding and addictive using of technological devices.

\section{References}

Acherer, R. F., Wiebe, F. A., Luther, D. C. \& Adams, J. S. (1988). Dimensionalty of coping: Factor stability using the ways of coping questionnaire. Psychology Reports, 62, 763-770.

Devellis, R. F., (2014). Scale Development, Theory and Applications. (Tarik Totan, Trans.), Ankara: Nobel Publishing.

Erkuş, A. (2012). Measurement and Scale Development in Psychology -I. Ankara: Pegem Academy.

Fried, D. (2014). Compulsive hoarding: Psychopathologies of print, phenomenologies of text. Culture, Theory and Critique, 55(3), 342-364.

Frost, R. O., \& Gross, R. C. (1993). The hoarding of possessions. Behaviour Research and Therapy, 31, $367-381$.

Frost, R., \& Hartl, T. (1996). A cognitive-behavioral model of compulsive hoarding. Behavior Research and Therapy, 34(4), 341-350.

Frost, R. O., Steketee, G., \& Grisham, J. (2004). Measurement of compulsive hoarding: saving inventory-revised. Behaviour research and therapy, 42(10), 1163-1182.

Frost, R. O., Steketee, G., Tolin, D. F., \& Renaud, S. (2008). Development and validation of the clutter image rating. Journal of Psychopathology and Behavioral Assessment, 30(3), 193-203.

Frost, R., Tolin, D., Steketee, G., Fitch, K., \& Selbo-Bruns, A. (2009). Excessive acquisition in hoarding. Journal of Anxiety Disorders, 23(5), 632-639.

Gormley, C. J., \& Gormley, S. J. (2012). Data hoarding and information clutter: The impact on cost, life span of data, effectiveness, sharing, productivity, and knowledge management culture. Issues in Information Systems, 13(2), 90-95.

Guillard, V., \& Pinson, C. (2012). Toward a better understanding and measurement of consumer hoarding. Recherche et Applications en Marketing, 27(3), 57-78.

Kaye, J., Vetis, J., Avery, A., Dafoe, A., David, S., Onaga, L., Rosero, I. \& Pinch, T. (2006). To have and to hold: exploring the personal archive. CHI Proceedings, Personal Information Management, April 22-27 2006, Montreal, $275-285$.

MacCallum, R. C., Widaman, K. F., Zhang, S. \& Hong, S. (1999). Sample size in factor analysis, Psychological Methods, 4(1), 84-99.

Özdamar, K. (2016). Scale and Test Development Structural Equation Modeling. Eskişehir: Nisan Bookstore.

Saxena, S., Brody, A., Maidment, K., \& Baxter, L. (2007). Paroxetine treatment of compulsive hoarding. Journal of Psychiatric Research, 41(6), 481-487.

Schneider, A., Storch, E., Geffken, G., Lack, C., \& Shytle, R. (2008). Psychometric properties of the Hoarding Assessment Scale in college students. Illness, Crisis, \& Loss, 16(3), 227-236.

Schüll, N. D. (2018). Digital containment and its discontents. History and Anthropology, 29(1), 42-48.

Sloane, S. (2011, 3). The problem with packrats: The high cost of digital hoarding. Forbes, 40.

Steketee, G., Frost, R., \& Kyrios, M. (2003). Cognitive aspects of compulsive hoarding. Cognitive Therapy and Research, 27(4), 463-479.

Stiff, R., Johnson, K. \& Tourk, K. A. (1975). Scarcity and hoarding: economic and social explanations and marketing implications. Advances in Consumer Research, 2(1), 203-16.

Sweeten, G., Sillence, E., \& Neave, N. (2018). Digital hoarding behaviours: Underlying motivations and potential negative consequences. Computers in Human Behavior, 85, 54-60.

Tavşancıl E. (2014). Measurement of Attitudes and Data Analysis with SPSS, 5th edn. Ankara: Nobel Publishing.

Tolin, D. F., Frost, R. O., \& Steketee, G. (2010). A brief interview for assessing compulsive hoarding: The Hoarding Rating Scale-Interview. Psychiatry Research, 178(1), 147-152. 
Tolin, D. F., Gilliam, C. M., Davis, E., Springer, K., Levy, H. C., Frost, R. O., ... \& Stevens, M. C. (2018). Psychometric properties of the Hoarding Rating Scale-Interview. Journal of Obsessive-Compulsive and Related Disorders, 16, 76-80.

Van Bennekom, M. J., Blom, R. M., Vulink, N., \& Denys, D. (2015). A case of digital hoarding. BMJ Case Reports 2015, 14.

Williams, P., Leighton John, J., \& Rowland, I. (2009). The personal curation of digital objects: A lifecycle approach. Aslib Proceedings, 61(4), 340-363.

Yurdugül, H. (2005). Use of scope validity indexes for scope validity in scale development studies. Pamukkale University Faculty of Education, XIVth National Educational Sciences Congress, 28-30 September, 771-774.

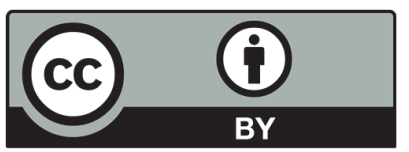

(C) 2020 by the authors; licensee Growing Science, Canada. This is an open access article distributed under the terms and conditions of the Creative Commons Attribution (CC-BY) license (http://creativecommons.org/licenses/by/4.0/). 